\title{
Type III CRISPR complexes from Thermus thermophilus
}

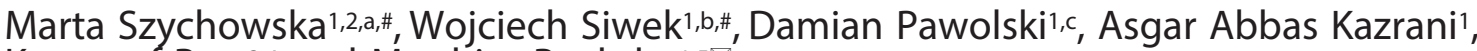 \\ Krzysztof Pyrc ${ }^{3,4}$ and Matthias Bochtler ${ }^{1,5 凶}$
}

IInternational Institute of Molecular and Cell Biology, Warsaw, Poland; 2Institute of Technical Biochemistry, Faculty of Biotechnology and Food Sciences, Technical University of Lodz, Łódź, Poland; ' ${ }^{M}$ Microbiology Department, Faculty of Biochemistry Biophysics and Biotechnology, JagielIonian University, Kraków, Poland; ${ }^{4}$ Malopolska Centre of Biotechnology, Jagiellonian University, Kraków, Poland; 5 Institute of Biochemistry and Biophysics PAS, Warsaw, Poland

Pathogen-specific acquired immunity in bacteria is mediated by the CRISPR (clustered regularly interspaced short palindromic repeats)-Cas systems. Thermus thermophilus strain HB8 contains CRISPR systems of several major subtypes (type I, IIIA and IIIB), and has become a widely studied model for CRISPR biology. We have selected two highly expressed CRISPR spacers, crRNA 2.1 and crRNA 2.2, and have enriched endogenous $T$. thermophilus proteins that co-purify with these crRNAs. Mass spectroscopy indicates that the chromatography protocol enriches predominantly Csm complex subunits, but also $\mathrm{Cmr}$ subunits. After several chromatographic steps, size exclusion chromatography indicated a molecular mass of the crRNA associated complex of $265 \pm 69 \mathrm{kDa}$. In agreement with earlier work, crRNAs of different lengths (containing the selected spacers) were observed. Most of these were completely lost when several $T$. thermophilus csm genes were ablated.

Key words: CRISPR-Cas, endonuclease, Csm, Cmr, Thermus thermophilus, crRNA, mass spectrometry (MS), chromatography

Received: 05 February, 2016; revised: 24 May, 2016; accepted: 24 May, 2016; available on-line: 14 June, 2016

\section{INTRODUCTION}

Pathogen-specific, acquired immunity against invading nucleic acids, like bacteriophages or plasmids, is mediated in bacteria by CRISPR-Cas systems (Marraffini \& Sontheimer, 2010a). These systems consist of the CRISPR loci and the CRISPR-associated (cas) genes (Wiedenheft et al., 2012; van der Oost et al., 2014). The CRISPR-loci store the immune memory in a form of pathogen-derived DNA sequences (known as spacers) separated by repeat sequences (Mojica et al., 2005; Barrangou et al., 2007). The Cas proteins cooperate with host factors to mediate spacer acquisition from invading DNA, crRNA biogenesis, and finally expression of immunity against invading DNA or RNA (Wiedenheft et al., 2012; van der Oost et al., 2014). Most bacteria harboring CRISPR-Cas systems share characteristic cas genes (typically cas1-casO) (Jansen et al., 2002a), which are complemented by additional, less widely distributed cas genes (Haft et al., 2005). The gene composition and architecture of the cas operons as well as the phylogenies of the most conserved and prevalent cas genes (cas1, cas 2 ) have been used to classify the CRISPR-Cas systems (Jansen et al., 2002a; Jansen et al., 2002b, Haft et al., 2005; Makarova et al., 2011). According to a recent review (Makarova et al., 2015), CRISPR systems come in two main classes.
Class 1 systems are defined by the presence of multisubunit crRNA effector complexes. They can be further classified into type I, type III and type IV, with characteristic signature genes (cas3 for type I, cas 10 for type III and csf1 for the poorly characterized type IV). Class 2 systems have a single subunit crRNA effector nuclease, either Cas9 for type II systems, or Cpf1 for type V systems. Type I and type II systems are already very well characterized (Jore et al., 2013; Zhao et al., 2014; Sinkunas et al., 2013; Gasiunas et al., 2012; Karvelis et al., 2013; Mali et al., 2013), not least because of the major role that Cas9 is now playing for genetic engineering applications (Mali et al., 2013; Jinek et al., 2012).

Type III CRISPR systems are broadly divided into type IIIA and type IIIB CRISPR systems, with Csm and $\mathrm{Cmr}$ effector complexes, respectively (depending on whether the signature gene is cmr1 or csm2). In vitro experiments with purified complexes initially pointed to an RNA endonucleolytic activity for both type IIIA (Tamulaitis et al., 2014; Staals et al., 2014) and type IIIB (Hale et al., 2009) complexes, but in vivo experiments on plasmid immunity were interpreted as evidence for an activity against invading DNA (Hatoum-Aslan et al., 2014; Marraffini \& Sontheimer, 2008). The puzzle was solved by the observation that plasmid immunity of Sulfolobus islandicus REY15A (a host of two different Cmr complexes) required transcription (Deng et al., 2013). Subsequently, it was shown that the Csm complex from Staphylococcus epidermidis also harbored a strictly transcription dependent DNA endonuclease activity (Samai et al., 2015). As the DNA and RNA endonuclease active sites were distinct, their roles could be genetically separated. Again using the $S$. epidermidis model, it was shown that the major contribution to plasmid and (DNA) virus immunity was made by the DNA endonucleolytic activity, with RNA endonucleolytic activity rather serving as a "backup" (Samai et al., 2015). Proto-spacer adjacent motifs are not required in targets of type III CRISPR systems. Instead, base pairing between crRNA and target extending beyond the spacer (or protospacer) region

e-mail: mbochtler@iiimcb.gov.pl

aCurrent address: Nencki Institute of Experimental Biology, Ludwika Pasteura 3, 02-093 Warsaw, Poland

bCurrent address: Instituto Gulbenkian de Ciência, 2780-156 Oeiras, Portugal

'Current address: Center for Molecular Bioengineering, Technische Universität Dresden, Arnoldstrasse 18, 01307 Dresden, Germany \#equal contribution

Abbreviations: CRISPR, clustered regularly interspaced short palindromic repeats; Cas, CRISPR-associated; crRNA, CRISPR-RNA 
and extending into the repeat region suppresses activity (Marraffini \& Sontheimer, 2010b).

The molecular architecture of type III CRISPR systems is largely conserved between type IIIA and IIIB systems and also reminiscent of the architecture of type I CRISPR complexes. A hallmark of all type III complexes is the occurrence of multiple copies of some of their subunits. Cryo-electron microscopy data for the Sulfolobus solfataricus and Thermus thermophilus Csm complexes, and for the Thermus thermophilus Cmr complex suggest a two-filament structure (Wiedenheft et al., 2011; Staals et al., 2013; Staals et al., 2014). The more prominent of these filaments serves as the primary binding site for the crRNA, the less prominent filament wraps around it. The remaining complex subunits "buttress" the filament pair at both ends. Biochemical data indicate substantial variability in the stoichiometry of the complexes, which appears to correlate with crRNA content.

The Csm complex from Staphylococcus epidermidis (which lacks other CRISPR-Cas systems simplifying the interpretation of in vivo results) (Marraffini \& Sontheimer, 2008; Marraffini \& Sontheimer, 2010b; Hatoum-Aslan et al., 2011; Hatoum-Aslan et al., 2014) has been described as a complex of $331 \mathrm{kDa}$ (Hatoum-Aslan et al., 2013). Formation of the complex was impeded in the absence of Csm1 (also known as Cas10), Csm3 or Csm4. However, subcomplexes containing all other members still formed when subunits Csm2 or Csm5 were missing (HatoumAslan et al., 2014). The Csm complex from S. solfataricus (which has several Csm3 paralogues) was described as a complex of $427 \mathrm{kDa}$, but several subcomplexes, most likely representing fragments of the full complex, were also observed (Rouillon et al., 2013). For the Streptococcus thermophilus Csm complex, masses of $345 \mathrm{kDa}$ and $486 \mathrm{kDa}$ were found, depending on whether the complexes were associated with short (40 nt) or long (72 nt) crRNAs (Tamulaitis et al., 2014). A Cmr complex from T. thermophilus was also described as having variable stoichiometry (molecular masses about 310 and about $350 \mathrm{kDa}$, bound crRNA of 40 or $46 \mathrm{nt}$ in length) (Staals et al., 2013).

T. thermophilus strain HB8 contains three cas operons of the type IE, IIIA and IIIB and twelve CRISPR loci, located both on the pTT27 megaplasmid and on the chromosomal DNA (see Fig. 1 in Staals et al., 2014). All cas operons are expressed, and the level of their transcription increases upon phage infection (Agari et al., 2009). In addition all CRISPR loci, except the CRISPR-8 locus, are constitutively transcribed and processed, and the pre-crRNAs are primarily cleaved 8 nt upstream of the spacer (Juranek et al., 2012). In this work, we have chosen two crRNAs (crRNA 2.1 and crRNA 2.2) which were previously reported to be highly abundant in T. thermophilus HB8 (Juranek et al., 2012) and purified protein complexes associated with them. We find that the mix of these two crRNAs is predominantly associated with $\mathrm{Csm}$, but also $\mathrm{Cmr}$ complexes. We determine the approximate mass of the complexes by size exclusion chromatography, and we demonstrate that the biogenesis or stability of these crRNAs is dependent on all tested Csm subunits and not only on Csm3 (the subunit recently shown to harbor the RNase activity).

\section{MATERIALS AND METHODS}

Bacterial strains and growth conditions. Wild type T. thermophilus strain HB8 (WT) (Oshima \& Imahori, 1974) and HB27 (lacking the CRISPR-2 array) (Henne et al., 2004) and $\Delta \operatorname{csm} 1, \Delta \operatorname{csm} 3, \Delta \operatorname{csm} 5$ strains were grown at $65^{\circ} \mathrm{C}$ in Thermus medium (TM, $0.8 \%$ polypeptone, $0.4 \%$ yeast extract, $0.2 \% \mathrm{NaCl}, \mathrm{pH} 7.0$ ) liquid culture shaken at $180 \mathrm{rpm}$ or on Thermus medium agar plates containing $1.5 \%(\mathrm{w} / \mathrm{v})$ agar. When required, TM was supplemented with kanamycin $(20 \mu \mathrm{g} / \mathrm{ml})$.

T. thermophilus transformation and gene deletion. T. thermophilus overnight culture was supplemented with $\mathrm{CaCl}_{2}$ and $\mathrm{MgCl}_{2}$ to the final concentration of $0.4 \mathrm{mM}$ and shaken at $65^{\circ} \mathrm{C}$ for $2 \mathrm{~h} .50 \mathrm{ml}$ of such culture was centrifuged and the pellet was suspended in $0.5 \mathrm{ml}$ of TM with $0.4 \mathrm{mM}$ of $\mathrm{CaCl}_{2}$ and $\mathrm{MgCl}_{2}$. The $\Delta \mathrm{csm} 1$, $\Delta \operatorname{csm} 3, \Delta \operatorname{csm} 5$ strains were made according to the protocol from the Thermus consortium (Hashimoto et al., 2001). One $\mu \mathrm{g}$ of deletion vector (commercially distributed by RIKEN consortium) was added to the suspension and the mixture was shaken at $65^{\circ} \mathrm{C}$ for $2 \mathrm{~h}$. Culture was then spread on TM agar plates supplemented with kanamycin and grown for $48 \mathrm{~h}$ at $65^{\circ} \mathrm{C}$.

Confirmation of gene deletion by Southern blot analysis. T. thermophilus megaplasmid DNA was isolated from $T$. thermophilus W'T and $\Delta \operatorname{csm} 1, \Delta \operatorname{csm} 3$, $\Delta \operatorname{csm} 5$ strains liquid cultures using Plasmid Midi AX kit (A\&A Biotechnology). Two $\mu \mathrm{g}$ of megaplasmid DNA from $\Delta$ csm1 strain was digested with R.SacI, digestion of megaplasmid DNA from $\Delta \mathrm{csm} 3$ and $\Delta \mathrm{csm} 5$ strains was performed with R.SmaI enzyme. The same restriction endonucleases were used to digest megaplasmid DNA from the WT strain as a negative control for the assay. Fragmented DNA samples were separated on $1 \%$ agarose gels alongside with a $\left[{ }^{33} \mathrm{P}\right]-5^{\prime}$-end radiolabeled GeneRuler DNA Ladder Mix (Thermo-Scientific). Gels were covered with denaturation buffer $(1.5 \mathrm{M}$ $\mathrm{NaCl}, 0.5 \mathrm{M} \mathrm{NaOH}$ ) and incubated at RT for $30 \mathrm{~min}-$ utes with gentle shaking. After removal of denaturation buffer gels were gently shaken with neutralization buffer (1 M Tris-Cl, $1.5 \mathrm{M} \mathrm{NaCl}, \mathrm{pH} 7.4$ ) at RT for $20 \mathrm{~min}-$ utes. DNAs were blotted to the positively charged nylon membrane (Ambion) by capillary transfer performed in $10 \times$ SSC for approximately $15 \mathrm{~h}$ and bound to the membrane by UV cross-linking. Prehybridization and hybridization was done at $42^{\circ} \mathrm{C}$ in the presence of prehybridization buffer ( $4 \times$ SSC, $0.5 \%$ SDS, $1 \times$ Denhardt's solution, $0.1 \mathrm{mg} / \mathrm{ml}$ herring sperm ssDNA). After $2 \mathrm{~h}$ of prehybridization, $\left[{ }^{32} \mathrm{P}\right]-5$ '-end radiolabeled probe specific for kanamycin resistance cassette (5'ATATATAGTGGATGTGTCAAAACGCATACCATTTTGAACGATGACCTCTAATAATTGTTAATCATGTTGGTTACGCTG-3') was added to the prehybridization buffer and hybridization was performed for at least $2 \mathrm{~h}$. Subsequently, the membrane was washed twice with washing solution ( $4 \times \mathrm{SSC}, 1 \% \mathrm{SDS})$ at $42^{\circ} \mathrm{C}$ for 20 minutes in order to remove unbound probe. The blots were overnight exposed to the phosphor screen and scanned using a STORM imager.

Confirmation of gene deletion by sequencing. T. thermophilus genomic DNA was isolated from $T$. thermophilus $\Delta \operatorname{csm} 1, \Delta \operatorname{csm} 3, \Delta \operatorname{csm} 5$ strains liquid cultures using Genomic Mini kit (A\&A Biotechnology). Amplification of $\mathrm{csm}$ loci was carried using genomic DNA from particular $T$. thermophilus deletion strains as a template. PCR reactions were performed in $50 \mu$ l of $1 \times$ DreamTaq green buffer containing $2 \mathrm{mM} \mathrm{MgCl}_{2}, 0.2 \mathrm{mM}$ of each dNTP, $0.5 \mu \mathrm{M}$ of relevant forward and reverse primers (Table 3), 1.25U DreamTaq DNA Polymerase, $50 \mathrm{ng}$ of genomic DNA. Touchdown PCR was performed with an initial denaturation step at $95^{\circ} \mathrm{C}$ for $3 \mathrm{~min}$ followed by 30 cycles, which involved a denaturation step at $95^{\circ} \mathrm{C}$ for $30 \mathrm{~s}$, annealing at $65^{\circ} \mathrm{C}$ for $30 \mathrm{~s}$ in the initial cycle and at decreasing temperatures by $0.5^{\circ} \mathrm{C} /$ cycle until 
a temperature of $50^{\circ} \mathrm{C}$ was reached, extension at $72^{\circ} \mathrm{C}$ for $1 \mathrm{~min}$. Final extension was performed at $72^{\circ} \mathrm{C}$ for $10 \mathrm{~min}$. PCR products were resolved on $1 \%$ agarose gels alongside with GeneRuler DNA Ladder Mix (ThermoScientific) and isolated from a gel using Gel/PCR Mini Kit (Syngen). Sequencing of PCR products was carried by Genomed as a commercial service. PCR products were sequenced using the same primers as for amplification of particular csm loci (Table 3).

Purification of crRNA-protein complexes. T. thermophilus HB8 cells were harvested by centrifugation. The pellet was suspended in $30 \mathrm{ml}$ of buffer $\mathrm{A}(5 \%(\mathrm{v} / \mathrm{v})$ glycerol, $50 \mathrm{mM}$ Hepes ( $\mathrm{pH}$ 8.0), $50 \mathrm{mM}$ NaCl, $10 \mathrm{mM}$ $\beta$-mercaptoethanol). Cells were disrupted in a French press at 40000 psi and the lysate was cleared by ultracentrifugation (20 min; $40000 \times g$ ). crRNA-protein complexes were purified from the cell extract by a combination of ion-exchange, affinity and size-exclusion chromatographic steps. After each step the presence of crRNA was tested by the Northern blot analysis and fractions containing crRNA were subjected to further purification steps. First, cell extract was applied on a HiTrap SP HP column (GE Healthcare Life Sciences) equilibrated with buffer A and flow-through was collected. The flow-through was then loaded on a HiTrap Q HP column (GE Healthcare Life Sciences) equilibrated with buffer A. The column was then washed with buffer $\mathrm{A}$ and proteins were eluted using $10-100 \%$ buffer B (5\% (v/v) glycerol, $50 \mathrm{mM}$ Hepes (pH 8.0), $1 \mathrm{M} \mathrm{NaCl}$, $10 \mathrm{mM} \beta$-mercaptoethanol) gradient in buffer A. Fractions that eluted at a conductivity between $14-23 \mathrm{mS} /$ $\mathrm{cm}$ were collected and buffer A was added to the sample to bring the final volume to $50 \mathrm{ml}$. Subsequently, sample was applied on HiTrap Heparin HP column equilibrated with buffer A. The column was then washed with buffer A. For the elution of proteins, a mix of $80 \%$ buffer A with $20 \%$ buffer $B$ was used, and the contribution of buffer B to the elution buffer was then gradually increased to $45 \%$. Fractions eluted at conductivity between $15-30 \mathrm{mS} / \mathrm{cm}$ were further purified with a flow rate of $0.5 \mathrm{ml} / \mathrm{min}$ on a HiPrep 16/60 Sephacryl S-400 HR column (GE Healthcare Life Sciences) equilibrated with buffer A. The volume of collected fractions was $2 \mathrm{ml}$. Proteins with retention volume in the range of 73-85 ml were pooled together and used for further analysis.

Preparation of samples for Northern blotting. T. thermophilus W'T and $\Delta \operatorname{csm} 1, \Delta \operatorname{csm} 3, \Delta \operatorname{csm} 5$ cells were harvested by centrifugation. The total RNA was isolated using TRIzol reagent (ThermoFisher) from approximately $100 \mathrm{mg}$ of the pellet. The RNA isolation was performed according to the protocol provided by the manufacturer. The concentration of RNA was measured on NanoDrop Spectrophotometer (Thermo-Scientific) and the amount of RNA in the sample was standardized (30 $\mu \mathrm{g}$ of RNA/sample). The samples were mixed with equal amount of formamide loading dye $(95 \%$ deionized formamide, $0.025 \%$ bromophenol blue, $5 \mathrm{mM}$ EDTA $\mathrm{pH}$ 8.0) and incubated at $95^{\circ} \mathrm{C}$ for 10 minutes. In the case of the fractions collected during purification of crRNA-protein complexes from T. thermophilus HB8 WT (including cell extract and samples collected after purification on HiTrap SP HP, HiTrap Q HP, HiTrap Heparin HP, HiPrep 16/60 Sephacryl S-400 HR columns) sample preparation was performed as follows. The extracts were concentrated on Vivaspin centrifugal concentrators (Sartorius) with $100 \mathrm{kDa}$ cut-off, standardized with regard to the total protein amount $(2000 \mu \mathrm{g}, 1500$ $\mu \mathrm{g}, 200 \mu \mathrm{g}, 50 \mu \mathrm{g}$ and $30 \mu \mathrm{g}$, respectively), mixed with
$15 \mu \mathrm{l}$ of formamide loading dye and incubated in $95^{\circ} \mathrm{C}$ for 10 minutes.

Northern blot detection of crRNAs. The RNA containing samples were separated alongside with a [33 P]-5'-end radiolabeled 10 bp DNA Ladder (Invitrogen) on $15 \%$ polyacrylamide $7 \mathrm{M}$ urea gels, transferred onto positively charged nylon membrane (Ambion) and immobilized on the membrane by UV cross-linking. Pre-hybridization and hybridization was performed at $42^{\circ} \mathrm{C}$ in the presence of pre-hybridization buffer $(4 \times \mathrm{SSC}$ buffer, $0.5 \%$ SDS, $1 \times$ Denhardt's solution, $0.1 \mathrm{mg} / \mathrm{ml}$ herring sperm ssDNA). After $2 \mathrm{~h}$ of prehybridization, $4 \mu \mathrm{l}$ of $\left[{ }^{32} \mathrm{P}\right]-5$ '-end radiolabeled probe (20 pmol of crRNA_2.1_probe: 5'-GAAAGAACATGCCGAAGCCGTCGTCGTTGGACT-3' and 20 pmol of crRNA_2.2_probe: 5'-CT'TCAGTCCCGGCCCGAGGCCGCTCACGCCCTC-3') or in case of the loading control with the $2 \mu$ of $\left[{ }^{33} \mathrm{P}\right]-5$ '-end radiolabeled probe against 5S rRNA (20 pmol of 5'-AGTACCATCGGCGCTGGCGCGT'T'TCACT'T-C-3' probe) or LeutRNA (20 pmol of 5'-TCAAGATGGCGTGTCTACCAGTTCCACCAC -3') was added to the pre-hybridization buffer and hybridization was performed for at least 2 h. Subsequently, the membrane was washed twice with washing solution $(4 \times \mathrm{SSC}, 1 \% \mathrm{SDS})$ at $42^{\circ} \mathrm{C}$ for $20 \mathrm{~min}-$ utes in order to remove unbound probe. The blot was overnight exposed to the phosphor screen and scanned using the STORM instrument.

Estimation of the molecular mass of the crRNA protein complex. The HiPrep 16/60 Sephacryl S-400 HR column was calibrated using thyroglobulin (670 $\mathrm{kDa}), \gamma$-globulin $(158 \mathrm{kDa})$, albumin fraction $\mathrm{V}(69 \mathrm{kDa})$ and myoglobulin $(17 \mathrm{kDa})$ as the molecular mass markers. Column calibration was done at a flow rate of 0.5 $\mathrm{ml}$. The retention volume of the crRNA-protein complex (after the HiTrap SP HP, HiTrap Q HP, HiTrap Heparin HP, HiPrep 16/60 Sephacryl S-400 HR purification steps) was verified by Northern blotting against crRNA 2.1 and crRNA 2.2 probe and used to estimate the molecular mass of the complex. Its error was deduced from the error of its logarithm, which in turn was estimated from the error of the retention volume and uncertainty of the calibration curve.

Proteomic analyses - MS and protein identification. Proteins co-purifying with crRNAs were analyzed by liquid chromatography coupled to the mass spectrometer in the Laboratory of Mass Spectrometry, IBB PAS (Warsaw, Poland). Samples were subjected to standard procedure of trypsin digestion, during which proteins were reduced with $10 \mathrm{mM}$ DT'T for 30 minutes at $56^{\circ} \mathrm{C}$ and alkylated with iodoacetamide in darkness for 45 minutes at room temperature and digested overnight with $10 \mathrm{ng} / \mathrm{ul}$ trypsin. The resulting peptide mixtures were concentrated and desalted on a RP-C18 pre-column (Waters), and further peptide separation was achieved on a nano-UPLC RP-C18 column (Waters, BEH130 C18 column, $75 \mu \mathrm{m}$ i.d., $250 \mathrm{~mm}$ long) of a nanoACQUITY UPLC system, using a 45 minutes linear acetonitrile gradient. Column outlet was directly coupled to the Electrospray ionization (ESI) ion source of the Orbitrap Velos type mass spectrometer (Thermo), working in the regime of data dependent MS to MS/MS switch. An electrospray voltage of $1.5 \mathrm{kV}$ was used. Raw data files were pre-processed with Mascot Distiller software (version 2.4.2.0, MatrixScience). The obtained peptide masses and fragmentation spectra were matched to the NCBI nonredundant database (57412064 sequences/20591031683 residues), with a Thermus thermophilus filter (10650 se- 
A

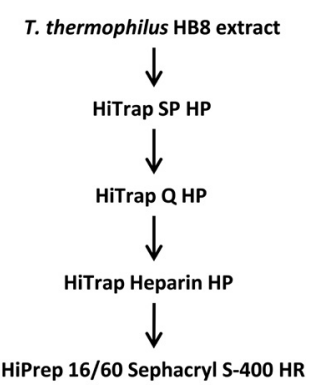

B

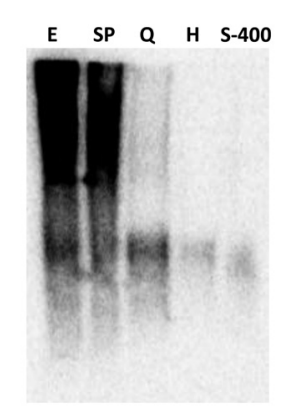

Figure 1. Purification scheme.

(A) Enrichment scheme for crRNAs from the CRISPR-2 locus and bound proteins. After each chromatography step, eluted fractions were analyzed by Northern blotting. A mixture of [32P]-5'-end labeled oligonucleotides serving as probes for crRNA 2.1 and crRNA 2.2 was used. Fractions with a strong Northern signal were pooled and used in the subsequent purification step. (B) Northern blot signal for the crRNAs present in T. thermophilus HB8 cell extract (E) and in the fractions collected after purification on the HiTrap SP HP (SP), HiTrap Q HP (Q), HiTrap Heparin HP (H) and HiPrep 16/60 Sephacryl S-400 HR column (S-400).

quences) using the Mascot search engine (Mascot Daemon v. 2.4.0, Mascot Server v. 2.4.1, MatrixScience). The following search parameters were applied: enzyme specificity was set to trypsin, peptide mass tolerance to $\pm 30 \mathrm{ppm}$ and fragment mass tolerance to $\pm 0.6 \mathrm{Da}$. The protein mass was left as unrestricted, and mass values as monoisotopic with one missed cleavage being allowed. Alkylation of cysteine by carbamidomethylation was set as fixed, and oxidation of methionine and carboxymethylation of lysine were set as variable modifications.

Protein identification was performed using the Mascot search engine (MatrixScience), with the probability based algorithm. Data were searched with automatic decoy database and were filtered to obtain a false discovery rate below $1 \%$.

DNA cleavage assays. The DNase activity of the sample obtained after all chromatographic steps was tested on a set of $5^{\prime}$ radio-labeled oligonucleotides ( $\mathrm{Ta}$ ble 2). The oligonucleotides were used at a final concentration of $0.005 \mathrm{pmol} / \mu \mathrm{l}$. The cleavage reactions contained $4 \mu \mathrm{l}$ of the purified sample with a protein concentration of $0.2 \mathrm{mg} / \mathrm{ml}$. The control samples contained water instead of the purified cell extract. The assays were performed for $1 \mathrm{~h}$ at $65^{\circ} \mathrm{C}$ in $20 \mu \mathrm{l}$ reaction volume with the standard FastDigest buffer (ThermoScientific) supplemented with $0.5 \mathrm{mM}$ ATP and $0.5 \mathrm{mM}$ GTP. Reactions were stopped by addition of $15 \mu$ l of formamide loading dye $(95 \%$ deionized formamide, $0.025 \%$ bromophenol blue, $5 \mathrm{mM}$ EDTA, $\mathrm{pH} 8.0$ ) and incubated at $95^{\circ} \mathrm{C}$ for 10 minutes. Reaction products were resolved on $15 \%$ polyacrylamide $7 \mathrm{M}$ urea gels alongside with a [ $\left.{ }^{32} \mathrm{P}\right]-5$ 'end radiolabeled GeneRuler Ultra Low Range DNA Ladder (ThermoScientific), transferred onto positively charged nylon membrane (Ambion) and UV crosslinked. The blot was exposed overnight to a phosphor screen and scanned using a STORM instrument.

\section{RESULTS}

\section{Chromatographic purification of crRNA-protein complexes}

crRNAs 2.1 and 2.2 (from the cluster termed NC_006462_3 in the CRISPRdb (Grissa et al., 2007)
Table 1. Mass spectrometry (MS) results of Cas proteins co-purifying with mature crRNAs from the CRISPR-2 locus.

Fractions collected after Heparin and Sephacryl S-400 chromatographic steps were subjected to mass spectrometry. Cas proteins in the samples were ordered according to their Mascot scores, which indicate the quality of identification (a high score corresponds indicates a confident identification).

\begin{tabular}{lcc}
\hline Name & Position on the list & \multicolumn{2}{l}{ Score } \\
\hline Results after the heparin column & \\
\hline Csm1 & 57 & 261 \\
\hline Csm3 & 58 & 261 \\
\hline Csm4 & 135 & 58 \\
\hline Csm5 & 141 & 52 \\
\hline Csm2 & 143 & 50 \\
\hline Results after the S-400 column & \\
\hline Csm3 & 11 & 1018 \\
\hline Csm5 & 24 & 655 \\
\hline Csm1 & 32 & 362 \\
\hline Csm4 & 37 & 316 \\
\hline Cmr4 & 42 & 264 \\
\hline Csm2 & 48 & 195 \\
\hline Cmr3 & 56 & 148 \\
\hline Cmr6 & 65 & 111 \\
\hline Cmr2 & 66 & 108 \\
\hline Cas7 & 72 & 85 \\
\hline Cmr1 & 76 & 80 \\
Cmr5 & 77 & 79 \\
\hline
\end{tabular}

nomenclature) are abundant in T. thermophilus, and their abundance increases upon phage infection (Agari et al., 2009). We purified macromolecular complexes of these crRNAs, using a protocol similar to that previously reported for the Cmr complex from P. furiosus (Hale et al., 2009). The cell extracts from the WT T. thermophilus HB8 strain were fractionated on SP, Q, Heparin and Sephacryl S-400 columns. After each chromatographic step we monitored the presence of crRNA 2.1 and crRNA 2.2 by Northern blot analysis (Fig. 1A). To increase sensitivity of the Northern blot analysis we used probes against crRNAs 2.1 and 2.2 simultaneously. Fractions in which we detected mature crRNAs were subjected to further purification. The Northern blot results show that throughout the purification the mature crRNAs 2.1 and 2.2 were separated from the larger crRNA species (Fig. 1B).

\section{Determination of the complex composition by mass spectrometry}

In order to identify proteins which co-migrate with mature crRNAs we analyzed the samples obtained after Heparin and Sephacryl S-400 chromatographic steps by mass spectrometry (Table 1). The analysis performed after Heparin chromatographic step identified 187 proteins including Csm1-5. After the Sephacryl S-400 chromatographic step 117 proteins were detected, including the Csm1-5 and Cmr1-6 proteins. Importantly, the Csm proteins were substantially enriched in the sample collected after chromatography on Sephacryl S-400 column when 
A
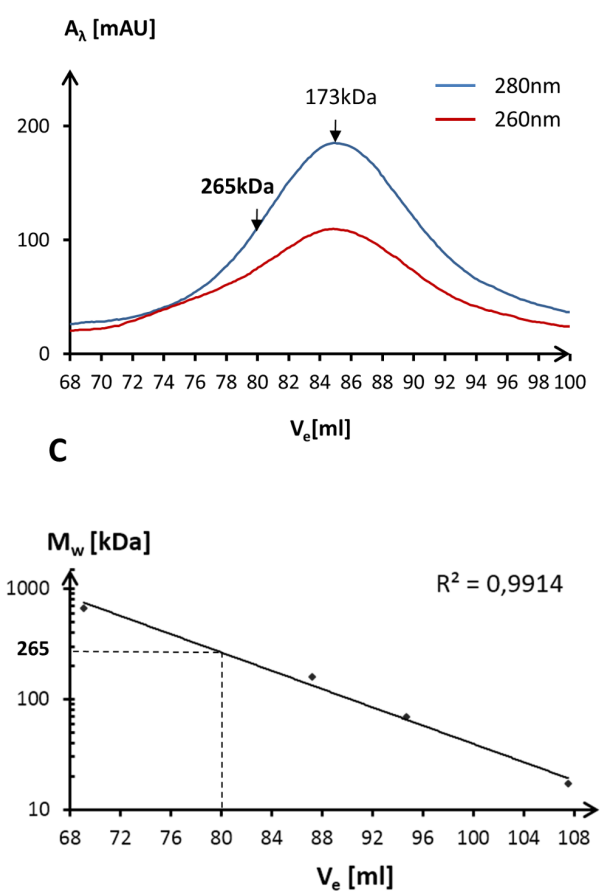

B

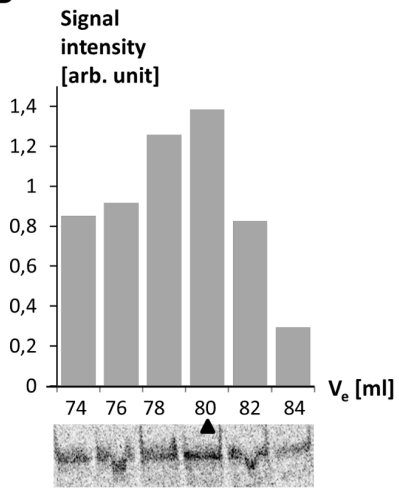

\begin{tabular}{|c|c|c|}
\hline Protein & $\mathrm{M}_{\mathrm{w}}[\mathrm{kDa}]$ & $\mathrm{V}_{\mathrm{e}}[\mathrm{ml}]$ \\
\hline Thyroglobulin & 670 & $69.1 \pm 0.4$ \\
\hline Y-globulin & 158 & $87.2 \pm 0.2$ \\
\hline Albumin fraction $\mathrm{V}$ & 69 & $94.7 \pm 0.4$ \\
\hline Myoglobulin & 17 & $107.5 \pm 0.6$ \\
\hline crRNA-complex & $\mathbf{2 6 5 \pm 6 9}$ & $80.0 \pm 2.0$ \\
\hline
\end{tabular}

Figure 2. Molecular sizing chromatography.

(A) The elution profile from the Sephacryl S-400 chromatographic column. Molecular mass estimates for the main peak (173 kDa) and for the peak of crRNA elution ( $265 \mathrm{kDa}$ ) are indicated. (B) crRNA elution profile analyzed by Northern blotting with the crRNA 2.1 and crRNA 2.2 probes. As the purification of the endogenous complex was only partial, the main peak of UV absorption (173 kDa) did not coincide with the peak for crRNA elution ( $265 \mathrm{kDa}$ ). The elution volume of mature crRNAs is indicated by an arrow. (C) Estimation of the crRNAcomplex molecular mass. The column was calibrated with the indicated set of molecular mass standards and the mass of the crRNA associated complex was obtained assuming a linear relationship between the molecular mass and the logarithm of the retention time. Based on the uncertainty of calibration line and the uncertainty of the retention time, the error of the mass is estimated to be $\pm 69 \mathrm{kDa}$. The molecular mass standards and the estimated mass of the crRNA-binding complex are indicated in the table.

compared to the sample obtained after purification on Heparin column (Table 1).

\section{Molecular mass estimation}

Next, we attempted to quantify the molecular mass of the crRNA associated complexes by quantifying the data from the size exclusion step (Fig. 2). As the native Csm and Cmr complexes appear to be present in T. thermophilus in low abundance, the peak of the UV absorption profile (presumably due to non-CRISPR associated proteins) was found at a lower mass than the maximum signal from Northern blotting, which was determined to correspond to a molecular mass of $(265 \pm 69) \mathrm{kDa}$.

\section{Activity assay}

Biochemical data available now demonstrate that the Csm complex targets DNA in a strictly transcription dependent manner (Konermann et al., 2015). Moreover, it can also cleave RNA (Tamulaitis et al., 2014, Staals et al., 2014). A protospacer adjacent motif (PAM) is not required (Marraffini \& Sontheimer, 2010b). At the time when the experiments described below were carried out, there was only genetic evidence for DNA targeting (Marraffini \& Sontheimer, 2008), but no in vitro demonstration of DNA or RNA endonuclease activity. In order to maximize the chances of observing cleavage in assays with single or double stranded DNA as the target, we chose flanking sequences for the protospacers that were previously shown to be present in an S. epider- midis Csm DNA substrate (in the two possible orientations, oligonucleotides assigned as $\mathrm{A}$ and B, Fig. 3). The control dsDNA and ssDNA oligonucleotides resembled

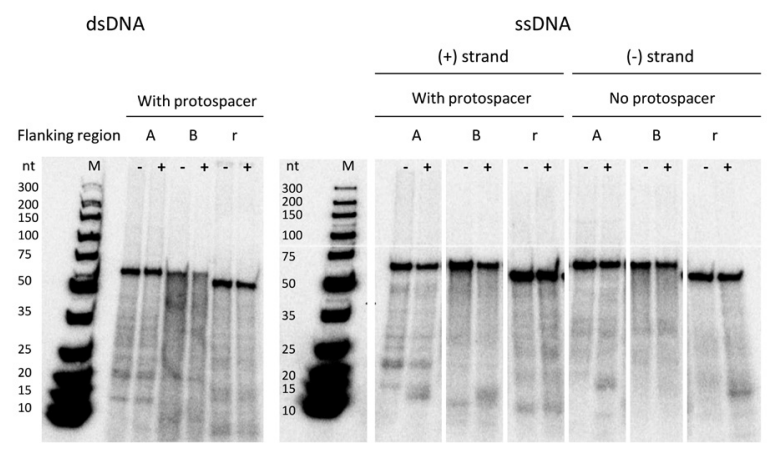

Figure 3. Autoradiogram demonstrating the inactivity of crRNA 2.1 associated complexes against DNA substrates.

The activity assay was performed on $5^{\prime}$-radiolabeled double stranded (dsDNA) and single stranded DNA molecules (ssDNA). Throughout, the extracts were tested after all four chromatography steps $(+)$, in comparison with the addition of no extract (-). Oligonucleotides designated with $\mathrm{A}$ and $\mathrm{B}$ letters are non-complementary outside the protospacer region and should be cleaved if the crRNA 2.1 associated complexes targeted DNA. All other DNA molecules were negative controls and should not be cleaved, either because they were complementary also to the repeat region (oligonucleotides designated with letter " $r$ "), or because their insert was identical to the crRNA spacer region rather than to the protospacer (no protospacer substrates). The full sequences of test substrates are given in Table 2. 
Table 2. DNA molecules used in the activity assay. The (+) strands of all oligodeoxynucleotides contain the region complementary to the crRNA 2.1 spacer sequence, the $(-)$ strands contain regions with sequence identical to the spacer (except for the obvious $U$ to $T$ change).

The spacer and protospacer sequences are indicated by upper case letters. In the control substrate protospacer flanking regions are complementary to the crRNA repeat region (DNA_r). The DNA_A and DNA_B substrates contain non-matching flanks. Although it was not expected that type III systems would require a PAM, we chose these regions identical to S. epidermidis protospacer flanking regions in two possible orientations (DNA_A and DNA_B). For comparison, the sequence of crRNA 2.1 is also given at the top of the table.

\begin{tabular}{|c|c|c|c|}
\hline & Strand & Sequence & Length \\
\hline crRNA 2.1 & & 5' - auugcgacAUGUGGCAGUCCAACGACGACGGCUUCGGCAUGUUCUUUCGguugc-3' & $54 \mathrm{nt}$ \\
\hline \multirow{2}{*}{ DNA_A } & $(+)$ & 5' -gattcttacCCGAAAGAACATGCCGAAGCCGTCGTCGTTGGACTGCCACATtctctaaata-3' & $61 \mathrm{nt}$ \\
\hline & $(-)$ & 5' -tatttagagaATGTGGCAGTCCAACGACGACGGCTTCGGCATGTTCTTTCGggtaagaatc-3' & $61 \mathrm{nt}$ \\
\hline \multirow{2}{*}{ DNA_B } & $(+)$ & 5' -tatttagagaCGAAAGAACATGCCGAAGCCGTCGTCGTTGGACTGCCACATggtaagaatc-3' & $61 \mathrm{nt}$ \\
\hline & $(-)$ & 5' -gattcttacCATGTGGCAGTCCAACGACGACGGCTTCGGCATGTTCTTTCGtctctaaata-3' & $61 \mathrm{nt}$ \\
\hline \multirow{2}{*}{ DNA_r } & $(+)$ & $5^{\prime}$-gcaacCGAAAGAACATGCCGAAGCCGTCGTCGTTGGACTGCCACATgtcgcaat-3' & $54 \mathrm{nt}$ \\
\hline & $(-)$ & $5^{\prime}$-attgcgacATGTGGCAGTCCAACGACGACGGCTTCGGCATGTTCTTTCGgttgc-3' & $54 \mathrm{nt}$ \\
\hline
\end{tabular}

the T. thermophilus genomic CRISPR array and contained the protospacer flanked by a part of the genomic repeat sequence (oligonucleotides assigned as " $r$ ", Fig. 3). In the cleavage assay on the ssDNA targets we used the oligonucleotides with sequence identical to the guide crRNA spacer as an additional control (oligonucleotides assigned as "(-) strand"). Since the CRISPR-Cas effector complexes mediate cleavage only if the target is complementary to the guide crRNA such oligonucleotides should not be cleaved. All DNA molecules were then end-labeled radioactively and then incubated with "+" or without "-" crRNA 2.1 associated complexes. No difference was observed (Fig. 3), in agreement with the current view that type IIIA and most likely also type IIIB complexes strictly require transcription of their DNA substrates (Samai et al., 2015).

\section{Gene disruption studies}

Next, we tested the effect of csm gene deletions on the content of crRNA in T. thermophilus extracts. Single csm gene deletions were carried out using gene disruption plasmids available from RIKEN (for $\operatorname{csm} 1, \operatorname{csm} 3$ and $\operatorname{csm} 5$ ). These plasmids carry kanamycin resistance genes for selection in $T$. thermophilus. They can replicate in E. coli, but not T. thermophilus, and therefore require an insertion in the latter bacteria to mediate resistance. Flanking regions target the resistance gene to the desired locus. As the resistance gene insertion is not "scar-less", only single gene mutations were constructed. Kanamycin resistance cassette insertion into the $\operatorname{csm} 1, \operatorname{csm} 3$ and $\operatorname{csm} 5$ was confirmed by Southern blotting (Fig. 4) and DNA sequencing (Table 3). We then probed the crRNA content of the cell extracts (and of extracts of T. thermophilus HB27, which lacks the CRISPR-2 array) by Northern blotting with the crRNA 2.1 and 2.2 probes. In contrast to the protein purification experiment, RNAs were pre-purified by the TRIzol method, which led to much sharper RNA bands, but also to a loss of hybridization signal already in the wild-type (Fig. 5).

In the wild-type, the majority of crRNAs 2.1 and 2.2 consisted of between $\sim 45$ and $\sim 70$ nucleotides, but there were also bands corresponding to smaller RNA molecules (Fig. 5). Our data do not have sufficient resolution to determine the precise size difference between these bands, the differences may correspond to the 6 nucleotide spacing between crRNA lengths found earlier for the crRNAs of S. epidermidis (Hatoum-Aslan et al., 2013) (and the 6 nucleotide spacing in RNA substrates reported recently) (Staals et al., 2014; Gasiunas et

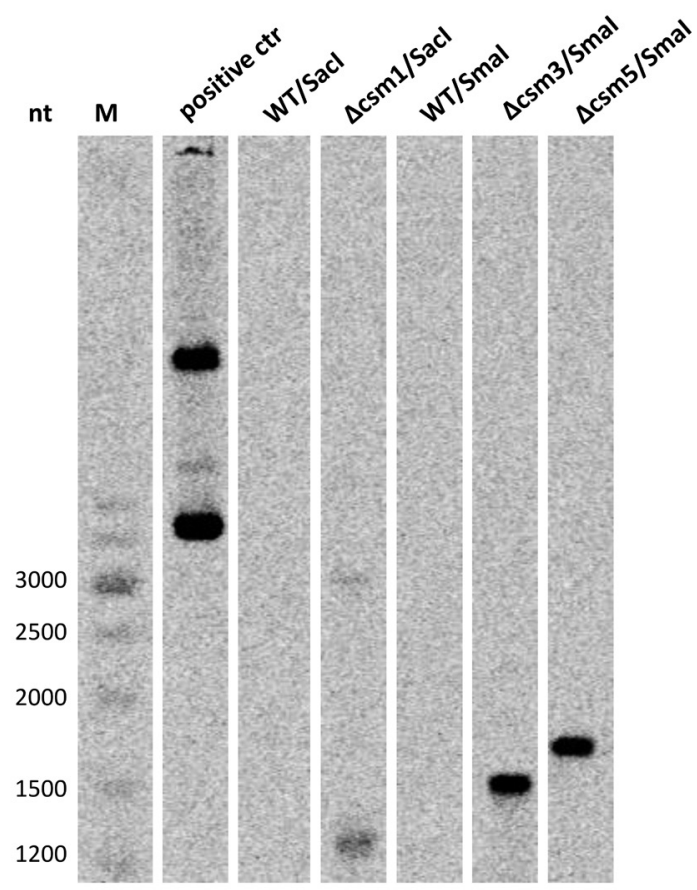

Figure 4. Confirmation of $\operatorname{csm} 1, \operatorname{csm} 3, \operatorname{csm} 5$ gene deletion by Southern blot analysis.

Single csm gene deletions were carried out by insertion of the kanamycin resistance cassette into targeted genes. The megaplasmid DNA isolated from T. thermophilus strains was digested with either R.Sacl or with R.Smal enzyme and tested for the presence of kanamycin resistance cassette. The kanamycin carrying genome fragments were expected to be 1344 (for the $\Delta$ csm1 strain), 1533 $(\Delta \operatorname{csm} 3$ strain) and $1715 \mathrm{nt}(\Delta \mathrm{csm} 5$ strain) base pairs long. Megaplasmid DNA from the wild type strain was used as a negative control. Positive ctr - csm1 disruption plasmid; $\mathrm{nt}$ - number of nucleotides; $\mathrm{M}$ - molecular mass marker. 


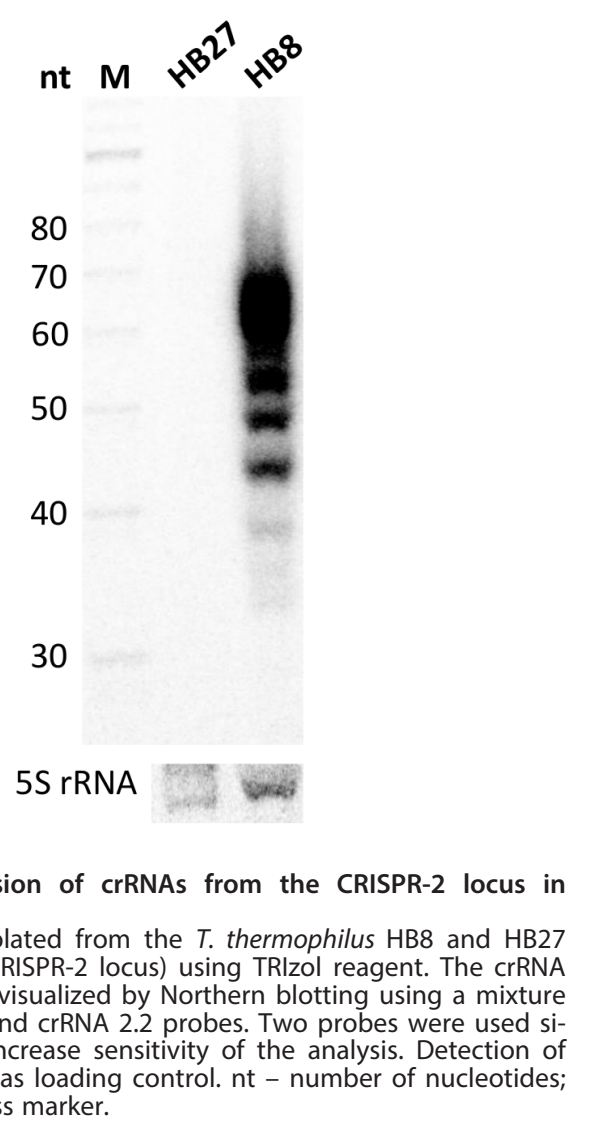

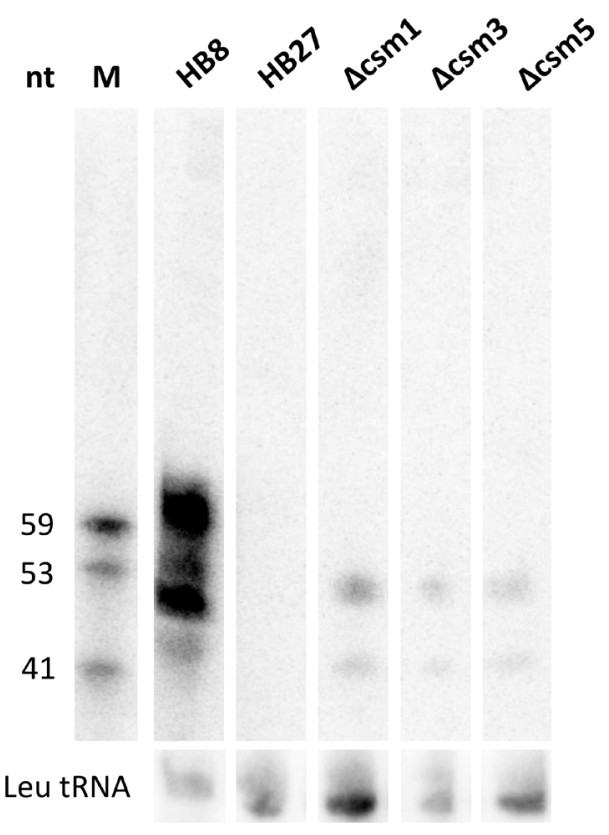

Figure 6. Effect of csm gene deletion on crRNAs from the CRISPR-2 locus in T. thermophilus.

Total RNA was isolated from the $T$. thermophilus HB8, HB27 and the otherwise isogenic $\Delta \operatorname{csm} 1, \Delta \operatorname{csm} 3, \Delta \operatorname{csm} 5$ strains using TRIzol reagent. The crRNA content was then visualized by Northern blotting using a mixture of the crRNA 2.1 and crRNA 2.2 probes. Two probes were used simultaneously to increase sensitivity of the analysis. Detection of Leu-tRNA is shown as loading control. nt - number of nucleotides; $\mathrm{M}$ - molecular mass marker.

Table 3. Confirmation of $\operatorname{csm} 1, \operatorname{csm} 3, \operatorname{csm} 5$ gene disruption by sequencing of relevant $\operatorname{csm}$ loci.

\begin{tabular}{|c|c|c|c|}
\hline $\begin{array}{l}\text { Disrupted } \\
\text { gene }\end{array}$ & \multicolumn{2}{|c|}{ Primer sequence } & Sequence of the relevant csm locus in the mutant strain \\
\hline \multirow{2}{*}{ csm1 } & $\mathrm{F}$ & $\begin{array}{l}\text { 5'GGGAGGTG- } \\
\text { CAGGTGCTGT- } \\
\text { GAG-3' }\end{array}$ & $\begin{array}{l}\text { 5' ATGCTTGGGGACGGCCTGAGCGTtctagacgttgacggcggatatggtacaatgaacatggagacatg } \\
\text { cagggttttcCtgcattttctttgtgcactcatcaagcgattttcggtaaagctaggaggaatga }\end{array}$ \\
\hline & $\mathrm{R}$ & $\begin{array}{l}5^{\prime} \text { GGGCCCGCT- } \\
\text { CAAAGACCT- } \\
\text { TGG-3' }\end{array}$ & $\begin{array}{l}\text { ggatgtgtcaaacgcataccattttgaacgatgacctctaataattgttaatcatgttggttacgctgc } \\
\text { agTTTACCGGGAAAGGAGGGTGTGA-3' }\end{array}$ \\
\hline \multirow[b]{2}{*}{ csm3 } & $\mathrm{F}$ & $\begin{array}{l}\text { 5'AGGCG- } \\
\text { GTGCTCGCC- } \\
\text { TACTTCTAC-3' }\end{array}$ & $\begin{array}{l}\text { 5' ATGAAGCTCAAGAAGGTGATCggtacccgttgacggcggatatggtacaatgaacatggagacatgca } \\
\text { gggttttcctgcattttctttgtgcactcatcaagcgattttcggtaaagctaggaggaaatgatg }\end{array}$ \\
\hline & $\mathrm{R}$ & $\begin{array}{l}5^{\prime} \text { GGGCCT- } \\
\text { TGGAAGTA- } \\
\text { GAGGCGGA- } \\
\text { AGAG-3' }\end{array}$ & $\begin{array}{l}\text { atgtgtcaaaacgcataccattttgaacgatgacctctaataattgttaatcatgttggttacgctgcag } \\
\text { GTGGAGGAAGTGGTCTTMA-3, }\end{array}$ \\
\hline \multirow[b]{2}{*}{ csm5 } & $\mathrm{F}$ & $\begin{array}{l}\text { 5'CACCGC- } \\
\text { CTGCTGGAG- } \\
\text { GTGAC-3' }\end{array}$ & $\begin{array}{l}\text { 5' GTGGGAGGCCCTCGTGGTCTTTCtctagacgttgacggcggatatggtacaatgaacatggagacatg } \\
\text { cagggttttcctgcattttctttgtgcactcatcaagcgattttcggtaaagctaggaggaatga }\end{array}$ \\
\hline & $\mathrm{R}$ & $\begin{array}{l}\text { 5'CCCGGTAC- } \\
\text { CGCTCCCA- } \\
\text { AAGGG-3' }\end{array}$ & $\begin{array}{l}\text { ggatgtgtcaaaacgcataccattttgaacgatgacctctaataattgttaatcatgttggttacgctgc } \\
\text { agTGGGGAGGCTAGAGCCCCCTAG3' }\end{array}$ \\
\hline
\end{tabular}

Confirmation of $\operatorname{csm} 1, \operatorname{csm} 3, \operatorname{csm} 5$ gene disruption by sequencing of the relevant csm loci. Single csm gene deletions were carried out by insertion of the kanamycin resistance cassette into targeted genes. The csm1, csm3, csm5 loci were amplified from genomic DNA of mutant Thermus thermophilus HB8 strains ( $\Delta \mathrm{csm} 1, \Delta \mathrm{csm} 3, \Delta \operatorname{csm} 5$, respectively) and sequenced. The primers used for amplification and sequencing of $c s m$ loci were designed to anneal in the regions flanking relevant csm genes. $F$ - forward primer; $R$ - reverse primer; upper case letters - fragments of the targeted gene left after gene disruption; lower case letters - kanamycin resistance cassette; black - region covered by sequencing; underlined region not covered by sequencing. Note that the csm5 open reading frame starts with GTG. The first few nucleotides in the kanamycin resistance gene in $\Delta \operatorname{csm} 3$ strains are different from those in the $\Delta \operatorname{csm} 1$ and $\Delta \operatorname{csm} 5$ strains, the reminders of the resistance genes are identical. 
al., 2012). As a result of the gene deletions, we observe a massive reduction in the amounts of the crRNA species (Fig. 6).

\section{DISCUSSION}

\section{Length distribution of crRNAs detected by crRNA 2.1 and 2.2 probes}

Sequencing studies of crRNAs from T. thermophilus extracts and crRNA fractions isolated from Csm and Cmr complexes show consistently that the precursor crRNAs are processed between the -8 and -9 positions in the repeat, so that mature crRNAs with a repeat derived 8 -nucleotide handle are formed. Despite the generality of this rule even for repeats of different types (which differ slightly in the repeat sequence even in this "handle" region), crRNAs bound to Csm complexes were found to have a variety of lengths, with some lengths (45 nucleotides, 53 nucleotides) represented more often than others (Staals et al., 2014). crRNAs bound to the Cmr protein complex were less diverse (Staals et al., 2013). In line with the latter result, we observe a defined set of crRNA lengths in our experiments that only probe crRNAs with two specific spacers from a single cluster, in agreement with earlier studies using a single spacer probe (see Fig. 6 of Juranek and coworkers (2012), the CRISPR cluster p'T27_3, referred to as cluster 2 in Staals and coworkers (2014)).

The CRISPR cluster 2 (pTT27_3) contains three repeats with two spacers only. The repeats are 36 nucleotides long, the spacers in between them have lengths of 41 and 40 nucleotides. A single cleavage at the -8 position of the repeat should therefore result in crRNAs of 77 and 76 nucleotides length. Complete removal of the repeat portion at the 3'-end of the crRNA would take off 28 nucleotides (36-8 nt for the 5'-handle), and hence result in crRNA lengths of 49 or 50 nucleotides. Any smaller crRNA must not only lack the repeat derived sequence at the 3 '-end, but also part of the spacer, or alternatively, must have an unusual 5'-end. With the pTT27_3.1 probe, Juranek and coworkers (2012) detect a faint crRNA Northern blot band at around $70 \mathrm{nt}$, and several additional bands between 40 and 60 nucleotides (Juranek et al., 2012). As in the previous work, we observe distinct bands, rather than a continuous size distribution. Assuming 8 nucleotides of repeat at the 5'-end, the main crRNA bands in the HB8 wild-type strain could result from slight to complete trimming of the repeat 3 '-ends. Some trimming into the spacer may occur as well, in line with conclusions for other type III systems (van der Oost et al., 2014) (Fig. 5).

\section{Effect of csm gene disruption on crRNAs 2.1 and 2.2}

The crRNA pool of $T$. thermophilus has been extensively characterized, but the proteins involved in their biogenesis have not been identified. In contrast, extensive work on crRNA biogenesis has been carried out for the type IIIA CRISPR system from Staphylococcus epidermidis (Hatoum-Aslan et al., 2011; Hatoum-Aslan et al., 2013; Hatoum-Aslan et al., 2014). These studies have shown that Cas 6 is responsible for the primary cleavage of crRNA but may require activation by Csm1 or Csm4 (Hatoum-Aslan et al., 2011; Hatoum-Aslan et al., 2014). The studies further showed that the primary processing product of crRNA is then trimmed further in a manner that requires a crRNA length measurement from the 5 '-end primary processing site (Hatoum-Aslan et al., 2011). Gene deletion experiments in S. epidermidis suggest that this step requires the $\operatorname{csm} 2, \operatorname{csm} 3$ and $\operatorname{csm} 5$ genes, because their ablation leads to accumulation of the primary processing product (Hatoum-Aslan et al., 2011). Further studies, again using $S$. epidermidis as the model, showed that the crRNA molecules generated by the primary cleavage were then processed further so that a series of RNA molecules differing between each other by six nucleotides in length were formed (Hatoum-Aslan et al., 2013). The correlation between the length of crRNA and the number of bound Csm3 molecules further suggested that a Csm3 "helix', now known to lie at the core of the Csm complex (Staals et al., 2014), binds crRNA in such a manner that each Csm3 subunit associates with 6 nucleotides of crRNA, which could explain the characteristic 6 nucleotide steps in crRNA sizes (irrespective of whether Csm3, now known to cleave target crRNA, or another nuclease is responsible for the post-processing of Cas6 cleaved crRNAs) (Hatoum-Aslan et al., 2013).

In T. thermophilus HB8, we observe a massive reduction in the amounts of all but two smaller RNA species upon deletion of the $\operatorname{csm} 1, \operatorname{csm} 3$ or $\operatorname{csm} 5$ genes. We suspect that type III CRISPR systems are not only involved in the post-processing of crRNAs, but also protect them from other RNases. Alternatively, it is also possible that insertion of the resistance cassette in some csm genes might affect the expression of the operon and thus of other csm genes. Two bands in the Northern blot persist at least to some extent in the absence of the csm genes, perhaps due to the presence of other CRISPR systems in T. thermophilus (which is not the case in S. epidermidis) (Fig. 6).

\section{Molecular mass of crRNAs 2.1 and 2.2 associated complexes}

In this work, we used an unbiased approach to purify proteins or protein complexes that are associated with the two crRNAs from a particular crRNA cluster (cluster 2 in the nomenclature of Staals et al.), which had previously been shown to be abundantly expressed. After three initial purification steps, we were able to detect by mass spectrometry all five Csm subunits (Csm1-5) with significant scores, and after an additional gel filtration step, we could detect both Csm and Cmr subunits in our preparations. The large improvement in the rank score of the peptides as well as the inclusion of the $\mathrm{Cmr}$ proteins most likely reflects the substantial enrichment by a chromatography step that excludes most T. thermophilus proteins that are smaller than the purified complexes. When this work was originally carried out, the crRNA bound to Csm and Cmr complexes of T. thermophilus had not yet been sequenced, and it was surprising that the crRNA was found to be associated with both complexes. However, it is now clear that crRNAs with spacers that are highly represented in Csm complexes are also abundant in Cmr complexes and vice versa. Hence, the detection of the crRNA 2.1 and 2.2 with both complexes is now expected. As Csm complexes seem to dominate over Cmr complexes in our enrichment scheme, the molecular mass determined by gel filtration should more closely match the T. thermophilus Csm mass than the reported Cmr masses.

The most recent mass estimate for the full Csm complex determined by mass spectrometry is about $427 \mathrm{kDa}$, but several smaller fragments of the complex were also detected (Staals et al., 2014). Our result, 265 09 kDa, is smaller than the previous value for the full complex 
determined by mass spectrometry. However, within error margin, our mass estimate for the $T$. thermophilus complex agrees with the mass of the Csm complex (331 kDa) (Hatoum-Aslan et al., 2013) from S. epidermidis, which also has one paralogue of the csm genes in its genome (unlike $S$. solfataricus). As in our case, the estimate for the $S$. epidermidis complex was made by gel filtration. It is therefore possible that gel filtration leads to systematically too small masses for the Csm complexes. However, we consider this unlikely because the Csm complexes are known to be elongated and therefore their apparent mass should exceed their theoretical mass. An alternative explanation could be that during the purification procedure, some more peripheral subunits may be lost. This explanation appears plausible in the light of the new structural data for the Csm complex (Staals et al., 2014) and need not contradict the observation that all subunits are detectable by the mass spectrometry, if the population of subcomplexes is heterogeneous. Another explanation for the smaller mass of the crRNA 2.1 and 2.2 associated complex compared to the reported mass for the T. thermophilus Csm complex could be a larger contribution from the $\mathrm{Cmr}$ complex than mass spectrometry suggests. The Cmr complex form T. thermophilus was previously found to be heterogeneous with molecular masses of 310 and $350 \mathrm{kDa}$, depending on the species of bound crRNA (Staals et al., 2013). At least the lower mass is compatible with the mass reported in this work.

\section{Activity of the complexes associated with crRNA 2.1}

The lack of activity of the purified fraction against DNA with crRNA 2.1 protospacers was surprising when the experiments were performed because genetic data suggested that the Csm complexes should target DNA. However, with the benefit of hindsight, the lack of activity against DNA substrates is now fully consistent with the recent discovery of strictly transcription dependent DNA cleavage activity of type III CRISPR systems (Konermann et al., 2015; Deng et al., 2013). The easiest explanation for transcription dependence of DNA cleavage by $\mathrm{Csm}$ (and possibly $\mathrm{Cmr}$ ) complexes appears to be that transcription generates single stranded DNA, which should anneal more easily with crRNA than double stranded DNA. However, experimental data by Samai and colleagues show that surprisingly, single stranded DNA is also not cleaved in the absence of transcription (Konermann et al., 2015), in agreement with the observations in this work. The reasons why single stranded DNA must be transcribed to be a substrate for type III CRISPR complexes remain to be understood.

\section{Acknowledgements}

We thank the Faculty of Biotechnology and Food Sciences of the Technical University of Lódź for allowing M.S. to carry out her M.Sc. thesis work at the International Institute of Molecular and Cell Biology. The RIKEN consortium is gratefully acknowledged for the csm gene deletion vectors. Prof. Michal Dadlez and his colleagues performed the mass spectrometry work as a commercial service. Dr. Ewa Sitkiewicz helped with the interpretation of the mass spectrometry results. We are grateful to Dr. Honorata Czapinska for critical reading of the manuscript.

This work was supported by grants N N302 654640 and UMO-2011/02/A/NZ1/00052 from the Polish National Science Centre to MB and UMO-2011/01/D/ NZ6/00269 to KP.

\section{REFERENCES}

Agari Y, Sakamoto K, Tamakoshi M, Oshima T, Kuramitsu S, Shinkai A (2009) Transcription profile of Thermus thermophilus CRISPR systems after phage infection. J Mol Biol 395: 270-281. doi: 10.1016/j. jmb.2009.10.057.

Barrangou R, Fremaux C, Deveau H, Richards M, Boyaval P, Moineau S, Romero DA, Horvath P (2007) CRISPR provides acquired resistance against viruses in prokaryotes. Science 315: 1709-1712. doi: $10.1126 /$ science. 1138140.

Deng L, Garrett RA, Shah SA, Peng X, She Q (2013) A novel interference mechanism by a type IIIB CRISPR-Cmr module in Sulfolobus. Mol Microbiol 87: 1088-1099. doi: 10.1111/mmi.12152.

Gasiunas G, Barrangou R, Horvath P, Siksnys V (2012) Cas9-crRNA ribonucleoprotein complex mediates specific DNA cleavage for adaptive immunity in bacteria. Proc Natl Acad Sci USA 109: E2579_ 2586. doi: $10.1073 /$ pnas.1208507109.

Grissa I, Vergnaud G, Pourcel C (2007) The CRISPRdb database and tools to display CRISPRs and to generate dictionaries of spacers and repeats. BMC Bioinformatics 8: 172. doi: 10.1186/1471-2105-8172.

Haft DH, Selengut J, Mongodin EF, Nelson KE (2005) A guild of 45 CRISPR-associated (Cas) protein families and multiple CRISPR/ Cas subtypes exist in prokaryotic genomes. PLoS Comput Biol 1: e60. doi: 10.1371/journal.pcbi.0010060.

Hale CR, Zhao P, Olson S, Duff MO, Graveley BR, Wells L, Terns RM, Terns MP (2009) RNA-guided RNA cleavage by a CRISPR RNA-Cas protein complex. Cell 139: 945-956. doi: 10.1016/j. cell.2009.07.040.

Hashimoto Y, Yano T, Kuramitsu S, Kagamiyama H (2001) Disruption of Thermus thermophilus genes by homologous recombination using a thermostable kanamycin-resistant marker. FEBS Lett 506: 231-234. doi: 10.1016/S0014-5793(01)02926-X.

Hatoum-Aslan A, Maniv I, Marraffini LA (2011) Mature clustered, regularly interspaced, short palindromic repeats RNA (crRNA) length is measured by a ruler mechanism anchored at the precursor processing site. Proc Natl Acad Sci USA 108: 21218-21222. doi: 10.1073/pnas.1112832108.

Hatoum-Aslan A, Samai P, Maniv I, Jiang W, Marraffini LA (2013) A ruler protein in a complex for antiviral defense determines the length of small interfering CRISPR RNAs. J Biol Chem 288: $27888-$ 27897. doi: 10.1074/jbc.M113.499244.

Hatoum-Aslan A, Maniv I, Samai P, Marraffini LA (2014) Genetic characterization of antiplasmid immunity through a type IIIA CRISPR-Cas system. J Bacteriol 196: 310-317. doi: 10.1128/ JB.01130-13.

Henne A, Bruggemann H, Raasch C, Wiezer A, Hartsch T, Liesegang H, Johann A, Lienard T, Gohl O, Martinez-Arias R, Jacobi C, Starkuviene V, Schlenczeck S, Dencker S, Huber R, Klenk HP, Kramer W, Merkl R, Gottschalk G, Fritz HJ (2004) The genome sequence of the extreme thermophile Thermus thermophilus. Nat Biotechnol 22: 547-553. doi: 10.1038/nbt956.

Jansen R, Embden JD, Gaastra W, Schouls LM (2002a) Identification of genes that are associated with DNA repeats in prokaryotes. Molecular microbiology 43: 1565-1575. doi: 10.1046/j.1365-2958.2002.02839.

Jansen R, van Embden JD, Gaastra W, Schouls LM (2002b) Identification of a novel family of sequence repeats among prokaryotes. OMICS 6: 23-33. doi: 10.1089/15362310252780816.

Jinek M, Chylinski K, Fonfara I, Hauer M, Doudna JA, Charpentier E (2012) A programmable dual-RNA-guided DNA endonuclease in adaptive bacterial immunity. Science 337: 816-821. doi: 10.1126/science. 1225829

Jore MM, Lundgren M, van Duijn E, Bultema JB, Westra ER, Waghmare SP, Wiedenheft B, Pul U, Wurm R, Wagner R, Beijer MR, Barendregt A, Zhou K, Snijders AP, Dickman MJ, Doudna JA, Boekema EJ, Heck AJ, van der Oost J, Brouns SJ (2013) Structural basis for CRISPR RNA-guided DNA recognition by Cascade. Nat Struct Mol Biol 18: 529-536. doi: 10.1038/nsmb.2019.

Juranek S, Eban T, Altuvia Y, Brown M, Morozov P, Tuschl T, Margalit H (2012) A genome-wide view of the expression and processing patterns of Thermus thermophilus HB8 CRISPR RNAs. RNA 18: 783-794. doi: 10.1261/rna.031468.111.

Karvelis T, Gasiunas G, Miksys A, Barrangou R, Horvath P, Siksnys $\mathrm{V}$ (2013) crRNA and tracrRNA guide Cas9-mediated DNA interference in Streptococcus thermophilus. RNA Biol 10: 841-851. doi: $10.4161 /$ rna.24203.

Konermann S, Brigham MD, Trevino AE, Joung J, Abudayyeh OO, Barcena C, Hsu PD, Habib N, Gootenberg JS, Nishimasu H, Nureki O, Zhang F (2015) Genome-scale transcriptional activation by an engineered CRISPR-Cas9 complex. Nature 517: 583-588. doi: $10.1038 /$ nature 14136.

Makarova KS, Haft DH, Barrangou R, Brouns SJ, Charpentier E, Horvath P, Moineau S, Mojica FJ, Wolf YI, Yakunin AF, van der Oost J, Koonin EV (2011) Evolution and classification of the CRISPR-Cas systems. Nat Rev Microbiol 9: 467-477. doi: 10.1038/ nrmicro 2577 . 
Makarova KS, Wolf YI, Alkhnbashi OS, Costa F, Shah SA, Saunders SJ, Barrangou R, Brouns SJ, Charpentier E, Haft DH, Horvath P, Moineau S, Mojica FJ, Terns RM, Terns MP, White MF, Yakunin AF, Garrett RA, van der Oost J, Backofen R, Koonin EV (2015) An updated evolutionary classification of CRISPR-Cas systems. Nat Rev Microbiol 13: 722-736. doi: 10.1038/nrmicro3569.

Mali P, Yang L, Esvelt KM, Aach J, Guell M, DiCarlo JE, Norville JE, Church GM (2013) RNA-guided human genome engineering via Cas9. Science 339: 823-826. doi: 10.1126/science.1232033.

Marraffini LA, Sontheimer EJ (2008) CRISPR interference limits horizontal gene transfer in staphylococci by targeting DNA. Science 322: 1843-1845. doi: 10.1126/science.1165771.

Marraffini LA, Sontheimer EJ (2010a) CRISPR interference: RNA-directed adaptive immunity in bacteria and archaea. Nat Rev Genet 11: 181-190. doi: 10.1038/nrg2749.

Marraffini LA, Sontheimer EJ (2010b) Self versus non-self discrimination during CRISPR RNA-directed immunity. Nature 463: 568-571. doi: $10.1038 /$ nature 08703 .

Mojica FJ, Diez-Villasenor C, Garcia-Martinez J, Soria E (2005) Intervening sequences of regularly spaced prokaryotic repeats derive from foreign genetic elements. J Mol Evol 60: 174-182. doi: 10.1007/s00239-004-0046-3

Oshima T, Imahori K (1974) Description of Thermus thermophilus (Yoshida and Oshima) comb. nov., a nonsporulating thermophilic bacterium from a Japanese thermal spa. Int J Syst Bacteriol 24: 102 112. doi: 10.1099/00207713-24-1-102.

Rouillon C, Zhou M, Zhang J, Politis A, Beilsten-Edmands V, Cannone G, Graham S, Robinson CV, Spagnolo L, White MF (2013) Structure of the CRISPR interference complex CSM reveals key similarities with cascade. Mol Cell 52: 124-134. doi: 10.1016/j.molcel.2013.08.020.

Samai P, Pyenson N, Jiang W, Goldberg GW, Hatoum-Aslan A, Marraffini LA (2015) Co-transcriptional DNA and RNA Cleavage during Type III CRISPR-Cas Immunity. Cell 161: 1164-1174. doi: 10.1016/j.cell.2015.04.027.
Sinkunas T, Gasiunas G, Waghmare SP, Dickman MJ, Barrangou R, Horvath P, Siksnys V (2013) In vitro reconstitution of Cascademediated CRISPR immunity in Streptococcus thermophilus. EMBO J 32: 385-394. doi: 10.1038/emboj.2012.352.

Staals RH, Agari Y, Maki-Yonekura S, Zhu Y, Taylor DW, van Duijn E, Barendregt A, Vlot M, Koehorst JJ, Sakamoto K, Masuda A, Dohmae N, Schaap PJ, Doudna JA, Heck AJ, Yonekura K, van der Oost J, Shinkai A (2013) Structure and activity of the RNAtargeting Type III-B CRISPR-Cas complex of Thermus thermophilus. Mol Cell 52: 135-145. doi: 10.1016/j.molcel.2013.09.013.

Staals RH, Zhu Y, Taylor DW, Kornfeld JE, Sharma K, Barendregt A, Koehorst JJ, Vlot M, Neupane N, Varossieau K, Sakamoto K, Suzuki T, Dohmae N, Yokoyama S, Schaap PJ, Urlaub H, Heck AJ, Nogales E, Doudna JA, Shinkai A, van der Oost J (2014) RNA targeting by the type III-A CRISPR-Cas Csm complex of Thermus thermophilus. Mol Cell 56: 518-530. doi: 10.1016/j.molcel.2014.10.005.

Tamulaitis G, Kazlauskiene M, Manakova E, Venclovas C, Nwokeoji AO, Dickman MJ, Horvath P, Siksnys V (2014) Programmable RNA shredding by the type III-A CRISPR-Cas system of Streptococcus thermophilus. Mol Cell 56: 506-517. doi: 10.1016/j.molcel.2014.09.027.

van der Oost J, Westra ER, Jackson RN, Wiedenheft B (2014) Unravelling the structural and mechanistic basis of CRISPR-Cas systems. Nat Rev Microbiol 12: 479-492. doi: 10.1038/nrmicro3279.

Wiedenheft B, Lander GC, Zhou K, Jore MM, Brouns SJ, van der Oost J, Doudna JA, Nogales E (2011) Structures of the RNAguided surveillance complex from a bacterial immune system. Nature 477: 486-489. doi: 10.1038/nature10402.

Wiedenheft B, Sternberg SH, Doudna JA (2012) RNA-guided genetic silencing systems in bacteria and archaea. Nature 482: 331-338. doi: 10.1038 / nature10886.

Zhao H, Sheng G, Wang J, Wang M, Bunkoczi G, Gong W, Wei Z, Wang Y (2014) Crystal structure of the RNA-guided immune surveillance Cascade complex in Escherichia coli. Nature 515: 147-150. doi: $10.1038 /$ nature13733. 